NOTAS Y DISCUSIONES

\title{
Anarquismo y liberalismo
}

\author{
FELIPE AGUADO HERNANDEZ \\ Instituto de Bachillerato Herrera Oria
}

\section{El intento sanarquista" de recuperación del liberalismo}

Como es bien sabido, en los paises de capitalismo avanzado se ha intentado, en los últimos tiempos y por variados caminos, la recuperación, tanto teórica como práctica, de los principios del liberalismo. Las políticas económicas y sociales de la mayoría de los gobiernos europeos, incluso de los de carácter socialdemócrata, se orientan por esos derroteros. La fórmula mágica que define los objetivos de esas políticas es la de la "recuperación de la sociedad civilw. El clásico concepto del más rancio liberalismo, la "sociedad civil", ha vuelto a ser desempolvado para justificar la vuelta a políticas capitalistas pre-keinesianas.

El estado benefactor había invadido las más recónditas esferas de la propiedad, pero la última gran crisis puso en entredicho el desarrollismo de la posguerra y la sociedad del bienestar que genero. Los beneficios de ese desarrollo llegaron, en mayor o menor medida, a muchos ciudadanos del primer mundo, merced a ese estado benefactor que gestionó el desarrollo de la legislación laboral protectora del trabajador, seguros de enfermedad y paro, pensiones de jubilación, etc. Pero la úl- tima gran crisis económica pone en entredicho ese modelo político-económico. Los beneficios empresariales se reducen y quiebran muchas empresas; el paro aumenta enormemente; en cambio, la esperanza de vida se alarga. Así, los ingresos en los sistemas de seguridad social caen mientras que, contradictoriamente, sus servicios deben aumentar por el incremento de jubilados y parados. Unos años en esta dinámica bastan para llevar a la bancarrota a las instituciones de seguridad social. EI modelo de estado benefactor entra en crisis.

¿Cómo encajar teóricamente todo el proceso? La vía fácil es echar las culpas de la crisis económica y de la propia bancarrota del sistema de bienestar a la excesiva intromisión del estado que, supuestamente, se habría convertido en un voraz predador de la economía empresarial y en un exhaustivo regulador de la vida pública en todas sus dimensiones. ¿Solución? Poner al estado en su sitio; en el auténtico lugar del estado en la sociedad liberal, ampliamente sobrepasado en el presente siglo, muy especialmente desde la implantación generalizada del keinesismo. Así, se despliegan las banderas de la recuperación de la «economía de mercado" y la "sociedad civil», del "li- 
beralismon sin tapujos ni adjetivos. Es decir, hay que recortar la presión equilibradora del estado en el reparto de la renta y en la protección de los trabajadores, para volver a formulas de autonomia y autorregulacion del empresariado capitalista. Como consecuencia, decrecen las prestaciones de la seguridad social; la legislación laboral retrocede permitiendo el trabajo precario; la presión fiscal sobre el empresario se aligera; se reestructuran sectores enteros de la producción que, saneados con fondos públicos, se reprivatizan a continuación (es decir, se devuelven a la "sociedad civil").

En este marco, ampliamente difundido, de crítica al estado benefactor y de recuperación de la sociedad civil, de vuelta al liberalismo en suma, algunos han intentado tender puentes hacia el anarquismo, en unos singulares intentos de recuperación del liberalismo. Acogidos tal vez a la profunda fobia anarquista por el estado y al amplio uso que hacen los libertarios de vocablos como libertad e individuo, esos intentos han querido encontrar amplios puntos de convergencia entre liberalismo y anarquismo, propugnando, a veces, incluso una especie de aliberalismo anarquista" o de "anarquismo liberaly.

$¿$ Es realmente coherente este tipo de propuestas? ¿Sobre qué base pueden descansar? Nuestro punto de vista es que no se puede hablar, si se pretende rigor conceptual e histórico, de convergencia anarquismo-liberalismo, al menos en referencia al anarquismo sindicalista de fin de siglo xIX y principios del $\mathrm{xx}$, que es el grueso de la tradición anarquista. Sin embargo, el anarquismo no es una doctrina monolftica $y$ hay planteamientos que se reclaman anarquistas fuera de la tradición señalada en los que tal vez sí podriamos encontrar bases antropologicas cerca- nas al liberalismo; por ejemplo, en los anarquistas stimerianos o en ciertas corrientes nietzscheanas. Veamos todo ello más despacio.

\section{Las ideas-base del anarquismo clasico}

El primer paso que hemos de dar es el de desbrozar el campo, delimitando lo que podemos entender por anarquismo para ahorrarnos confusiones. No cs esto nada fácil desde el momento en que los anarquistas, por principio, rechazan toda ortodoxia y toda doctrina fija e inamovible. Sin embargo, se pueden delimitar algunos planteamientos base que reconoce el grueso de la tradición anarquista y que pueden rastrearse en sus principales teóricos: Proudhon, Bakunin, Kropotkin, Malatesta. Podriamos citar tal vez como representativo de los lugares más comunes de esta tradición anarquista el "Catecismo Revolucionario" de Bakunin (1866). De él se pueden entresacar párrafos tan significativos como los siguientes:

IV. No es cierto que la libertad de un hombre esté limitada por la de los demás hombres. El hombre es realmente libre cuando su libertad, completamente reconocida por los demás y reflejada en ellos, encuentra su afirmación y su expansión on la libertad de los demás. El hombre no es realmente libre más que entre hombres igualmente libres; la esclavitud de un solo hombre ofende a la humanidad y niega la libertad de todos.

V. La libertad de cada uno, en consecuencia, sólo es posible mediante la igualdad de todos. La realización de la libertad por medio de la igualdad, en principio y de hecho, es justicia.

[...]

VII. Rechazo absoluto de cualquier autoridad incluyendo la que sacrifica la libertad por la conveniencia del Estado [...] A partir de ahora, el orden en la sociedad debe ser 
el resultado de la libertad individual mayor posible, así como de la libertad a todos los nivcles de la organización social.

VIII. La organización política y económica de la vida social no debe estar dirigida, como sucede ahora, de arriba abajo - del centro a la circunferencia- sino según el principio de asociaciones de federaciones libres.

DX. Organización polftica. Es imprescindible determinar una norma concreta, universal y obligatoria para el desarrollo interno y de la organización política de cada nación [...] No obstante, sin ciertas condiciones absolutamente esenciales, la realización de la libertad sería imposible para sicmpre. ${ }^{1}$

Esas condiciones serfan las siguientes:

Abolición de las clases, los rangos y los privilegios; absoluta igualdad de derechos políticos para todos los hombres y todas las mujeres: sufragio universal.

Abolición, disolución y desmantelamiento del Estado centralizado [...] Abolición de las universidades estatales: la educación pública debe ser administrada únicamente por las comunidades y asociaciones libres. Abolicion del poder estatal judicial; todos los jueces deben ser elegidos por el pueblo. Abolición de todos los códigos criminales, civiles y legales ahora vigentes en Europa: porque el código de la libertad sólo puede ser creado por la misma libertad. Abolición de toda administración centralizada, de la burocracia, de tados los ejércitos y policías estatales permanentes. ${ }^{2}$

\section{$Y$ más adelante:}

La libertad puede y debe defenderse únicamente mediante la libertad: proponer la restricción de la libertad con el pretexto de que se defiende es una peligrosa ilusión. ${ }^{3}$

En el plano economico, el anarquismo se declara enemigo de la propiedad privada y pretende su abolición. Sería sustituida por formas solidarias de tipo colectivista o comunista, punto en el que aparecen dos claras tendencias en el anarquismo evolucionado de fin del s. XIX y principios del XX. Si bien todos los anarquistas pretenden la sustitución del sistema capitalista por otro socialista de propiedad y gestión colectiva, unos se inclinan por que en esa nueva formación economica se retribuya a cada cual según su trabajo (colectivistas), y otros (comunistas) pretenden la utopía radical del «a cada cual según sus necesidades». Malatesta, en su diálogo "Entre Campesinos», escribe:

Los comunistas libertarios y los colectivistas son todos socialistas; pero tienen ideas diversas respecto a lo que debe hacerse después que la propiedad sea común. Los colectivistas dicen que cada trabajador, o mejor dicho cada asociación de trabajadores, debe poseer las materias primas y los instrumentos para trabajar, y cada uno debe ser duefo del producto de su trabajo [...] [los comunistas libertarios opinan que] cada individuo dará a la socjedad todo aquello que sus fuerzas le permitan dar [...] [y] cada uno tomara aquello que necesite, limitándose, se entiende, en todas aquellas cosas en las cuales no se haya podido llegar a la abundancia. ${ }^{4}$

\section{Algunos elementos de análisis comparativo anarquismo/tiberalismo}

De esta pequeña panorámica de puntos de vista anarquistas clásicos podemos concluir lo siguiente:

a) En primer lugar, se hace hasta cierto punto comprensible el intento del que se puede llamar aliberalismo anarquistan.

El rechazo absoluto del estado en todas sus formas por los anarquistas — El Estado es la negación de la humanidad", Bakunin-," podrfa interpretarse como paralclo a ciertas tesis liberales clásicas. Para los contractualistas, el individuo es anterior al esta- 
do; sólo aceptan éste como mal menor: preservación de los derechos fundamentales del individuo. Por ello el estado debe desempeñar unas funciones limitadas, subsidiarias de la «sociedad civil", en la que no debe injerirse, y estará siempre obligado a no lesionar o sustituir los derechos individuales.

Complementariamente, el rechazo anarquista del estado se hace en base a las exigencias de la realización en libertad del individuo, lo que también podría provocar simpatías liberales, dada la firme defensa de sus partidarios de las tesis de la igualdad y libertad de los individuos como núcleo de la sociedad civil y del estado.

b) Sin embargo, estas similitudes son claramente superficiales. Sólo un reflejo primario puede asimilar la defensa del individuo frente al estado de los anarquistas a las tesis liberales. Profundizar en ambos planteamientos revela las nitidas y abismales diferencias, no sólo de grado o matiz, sino de fundamento. Las tesis políticas y sus bases antropologicas son radicalmente opuestas en libertarios y liberales clásicos.

b.1) En primer lugar, el «individuo anarquistas no es el "individuo liberal". Este último es concebido como una isla que se fundamenta en sí misma y que construye la sociedad en función de su interés particular, centrado en la defensa de sus derechos como individuo, entre los que destaca, además de los derechos a la vida y la libertad, el derecho a la propiedad. Escribía Locke que lo que conduce al hombre "a unirse en sociedad con otros que ya están unidos [...] [es] el fin de preservar sus vidas, sus libertades y sus posesiones, es decir, todo eso a lo que doy el nombre genérico de "propiedad" ${ }^{6}$. La esencia del individuo liberal es la posesión. MacPherson describía muy plásticamente este carácter del liberalísmo cuando lo calificaba como aindividualismo posesivon. ${ }^{7} \mathrm{Y}$ no hay que recordar que el derecho liberal a la propiedad (posesión) incluye no sólo la de los bienes de consumo, sino también la de los bienes de producción. La igualdad liberal es la igualdad de los propietarios, patente en las teorias censatarias de los primeros teóricos liberales. La libertad de todo hombre, para los clásicos liberales, termina donde empiezan los derechos de los demás, entre los cuales ocupa un lugar destacado su propio derecho de propiedad o posesion; es decir, la libertad de los liberales es igualmente una libertad de propietarios.

Los teóricos anarquistas clásicos ya pusieron de manifiesto sus distancias respecto a las tesis liberales sobre el individuo. La antropologia anarquista destaca también el valor singular y absoluto del individuo. Pero no del individuo a-social de los liberales sino del individuo constitutivamente social que encuentra su desarrollo como singular en tanto se construye la solidaridad del colectivo humano. Bakunin comienza su Dios y el Estado precisamente con un análisis crítico de los adoctrinarios liberales». Reconoce que "son y se llaman liberales porque toman la libertad individual por base $y$ por punto de partida de su teorías. ${ }^{8}$ Pero, frente a su concepción individualista del hombre, Bakunin ofrece su concepción solidaria: «El hombre no se convierte en hombre y no llega, tanto a la conciencia como a la realización de su humanidad, más que en la sociedad y solamente por la acción colectiva entera»." En cualquiera de los teóricos libertarios clásicos encontramos formulas parecidas que expresan una concepción del hombre como constitutivamente social frente al a-social (por origen natural) hombre liberal. Por ejemplo, Enrico Malatesta: «Según mi modo de 
ver, los principios del individualismo son completamente erróneos. El individuo humano no es un ser independiente de la sociedad, sino su producto. Sin sociedad no habría podido salir de la esfera de la animalidad bratal y transformarse en un verdadero hombren. ${ }^{10} \mathrm{Y}$ frente a la concepcion liberal de la libertad intralimitada de los individuos-islas, Bakunin entiende que «no soy verdaderamente libre más que cuando todos los seres humanos que me rodean, hombres y mujeres, son igualmente libres. La libertad de otro, lejos de ser un límite o la negación de mi libertad, es al contrario su condicion necesaria y su confirmación»." $\mathrm{Y}$ Malatesta: «La libertad de cada uno no encuentra límite, sino el complemento y las condiciones necesarias a su existencia, en la libertad de los demás».12

b.2) En segundo lugar, el rechazo anarquista del estado no es homologable con la mera oposición liberal a las ex. tralimitaciones del estado. La postura libertaria ante el estado se funda en su concepción del hombre como ser solidario, claramente diferente de la mera oposición liberal a que el estado trascienda las funciones que, en su provecho, le encomienda el individuo posesivo. El liberalismo rechaza el estado sólo en tanto se inmiscuye en la «vida privadas de los individuos y se injiere en la "sociedad civil», pero acepta su función de marco legal del contrato comercial, así como su papel de defensor de los intereses económicos establecidos de los individuos propietarios. Escribia Locke: «El grande y principal fin que lleva a los hombres a unirse en Estados y a ponerse bajo un gobierno, es la preservación de su propiedad ${ }^{13}$ Es, pues, un estado hecho a imagen de la clase poseedora: potencia y defiende, juridica y físicamente, la posesión y no se injiere en la manera de gestionarla y aprovecharla. Pero, según los anarquistas, los teóricos liberales silencian precisamente la otra cara de su estado: la opresión sobre los no-poseedores. Así, el rechazo anarquista del estado es el rechazo de la autoridad, la fuerza y la prepotencia del estado frente a los débiles y el apoyo que presta a los intereses de los poseedores: «La crítica que los liberales hacen del gobierno se rcduce a despojarlo de un cierto número de atribuciones, pero no pueden atacar las funciones represivas que constituyen su esencia, por cuanto, sin gendarmes, el propietario no podría existir». ${ }^{14}$

Resumiendo. Hay ciertas aparentes similitudes entre liberalismo y anarquismo en cuanto a crítica del estado y defensa del individuo. Sin embargo son sólo aproximaciones superficiales, en cuanto que, profundizando, remiten a concepciones distintas $y$ aún contradictorias del hombre, la sociedad y el fundamento de las instituciones. Por ello no se sostiene ningún intento de "liberalismo anarquista" o eanarquismo liberal" sin distorsionar profundamente las tesis de uno o del otro.

Pero tales intentos de síntesis no son solo contemporáneos; los hubo en momentos pretéritos de mayor auge del anarquismo. Los propios Kropotkin y Malatesta salieron al paso de ellos. Unas líneas de Malatesta situan con gran claridad la cuestion: «El liberalismo viene a ser teóricamente una especie de anarquía sin socialismo, y por tanto no es sino mentira, un engaño, puesto que la libertad no puede existir $\sin$ la igualdad; la anarquía verdadera es inconcebible fuera de la solidaridad, fuera del socialismon. is No hay respuesta más contundente a intentos que, desde esta perspectiva, podríamos calificar como pseudoanarquizantes, de teóricos como R. Nozick y su «estado mínimo». ${ }^{16}$ En definitiva, es el "socialismo" lo que separa radical y abso- 
lutamente anarquismo y liberalismo. $Y$, desde este punto de vista, parece que no hay componendas posibles sin distorsionar gravemente el sentido histórico de las teorías y movimientos sociales libertarios.

\section{La impropiedad del «liberalismo anarquistas}

Sin embargo, como escribramos al principio de estas páginas, el anarquismo no es una ortodoxia monolítica. Hemos hablado del anarquismo sobre la base común de los más importantes y significativos de sus teóricos, reconocidos y admitidos como tales por el grueso de la tradición libertaria. No obstante, se han reclamado del anarquismo otros puntos de vista que, basándose en el absoluto del individuo, han desbordado las tesis societarias de los clásicos más admitidos. El caso más notorio es el de los stirnerianos, cuyos supuestos individualistas egoistas son similares y, a veces, la base de otras tendencias anarquistas individualistas tales como los nihilistas rusos, los nietzscheanos, los seguidores de $\mathrm{E}$. Armand, etc.

En lineas generales se puede afirmar que el llamado anarquismo individualista es una línea marginal respecto del anarquismo mayoritario. Sus tesis di- fieren de éste en que anteponen individuo a sociedad y, por tanto, egoŕsmo a solidaridad. Kropotkin analiza con claridad el fenómeno:

Stirner [...] no sólo invocaba una rebelión total contra el Estado y contra la servidumbre que el comunismo autoritario quisiera imponer a los hombres, sino también la completa liberacion del individuo respecto de todo vínculo social y moral - - la rehabilitación del «yos, la supremacía del individuo, el amoralismo total y la «asociación de egoístas». La conclusión final de este tipo de anarquismo individualista [...] no es de ninguna manera la posibilidad de que todos los miembros de la comunidad se desarrollen normalmente, sino la posibilidad de que ciertos individuos más dotados use desarrollen completamente", incluso a costa de la felicidad y de la misma existencia de la humanidad; se trata, pues, de la vuelta del individualismo más corriente. ${ }^{17}$

Lo que explica que muchos de sus partidarios «abandonen las filas del anarquismo y desemboquen en el individualismo liberals. ${ }^{18}$

Esta corriente marginal si daría pie a un enlace con el liberalismo sobre la base del común individualismo egoísta y a-social. Sin embargo, la propia exterioridad del stirnerismo y similares respecto del grueso de la tradicion anarquista harfa impropio un liberalismo que quisiera autoproclamarse "anarquista".

\section{NOTAS}

1. C. Díaz, Las teorias antarqutstas, Bilbao, Zero, pp. 63-64.

2. Toid., p. 64 .

3. Ibid., p. 65 .

4. E. Malatesta, «Entre campesinosn, en Sociatisno y anarquta, Madrid, Ayuso, 1975, pp. 113114.

5. M. Bakunin, Dios y el Estado, Buenos Aires, Proyección, 1971, p. 48 .

6. J. Locke, Segundo tratado sobre el gobiemo civil, Madrid, Alianza, cap. 9, n." 123.
7. C.B. MacPherson, La teoria poltica del individualisno posesivo, Barcelona, Fontanella, 1979. En el capftulo VI (pp. 225-226) de esta obra leemos lo siguiente:

"Los supuestos del individualismo posesivo pueden resumirse en las siete proposiciones siguientes:

I) Lo que hace humano a un hombre es ser libre de la dependencia de las voluntades de los demás.

II) La libertad de la dependencia de los demís 
significa libertad de cualquier relación con los demás salvo aquellas relaciones en las que el individuo entra voluntariamente por su proplo interess.

III) El individuo es esencialmente el propietario de su propia persona y de sus capacidades. por las cuales nada debe a la sociedad.

[...]

IV) Aunque el individuo no puede alienar toda su propiedad sobre su propia persona, puede alienar su capacidad para trabajar.

v) La sociedad humana consiste en una serie de relaciones mercantiles.

[...]

VI) Dado que lo que hace humano a un hombre es la libertad de las voluntades ajenas, la libertad de cada individuo solamente puede limitarse justamente por unas obligaciones y reglas tales que sean necesarias para garantizar la misma libertad a los demás.

VII) La sociedad polftica es una invención bumana para la protección de la propiedad que el individuo tiene sobre su propia persona y sobre sus bierses, y (por tanto) para el mantenimiento de relaciones de cambio debidamente ordenadas entre individuos considerados como propietarios.*

8. M. Bakunin, op, cit., p. 10 .

9. Ibid., p. 17.

10. E. Malatesta, «El individualismo en el anarquismon, en Socialismo y anarquia, op. cit., p. 58 .

11. M. Bakunin, op. cit. p. 18.

12. E. Malatesta, La anargufa, Bilbao, Zero, 1978, p. 50 .
13. J. Locke, op. cit., cap. $9, \mathbf{n}_{*}$ " 124.

14. E. Malatesta, La anarquia, op, cit., p. 76 .

15. Mbid, p. 76.

16. R. Nozick, Anarguía, Estado y utopia, Mexico, Fondo de Cultura Económica, 1988.

El autor, a lo largo de toda la obra, intenta convencemos de la necesidad, justicia y posibilidad de lo que él denomina astado mfnimon e incluso *ultrammimos que, en definitiva, no es otra cosa que el estado liberal, subsidiario de la sociedad civil en lo que los individuos y sus asociaciones elementales no pueden lograr por st mismos. Ese sestado mfnimos, por otra parte, pretende autonomizarlo de los restantes niveles sociales, en concreto del economico, en unos planteamientos tan absolutamente ingenuos que sorprende verlos reflejados en una obra que se pretende enjundiosa y que ha levantado ura gran expectacion. Transcribo un fragmento a modo de ilustracion: aAunque la estructura es libertaria y de laissez-faire, las conunidades individuales dentro de ella no necesitan ser ast $\mathrm{y}$, tal vez ninguna comunidad dentro de ella escoja ser asi. De esta manera, las características del marco no necesitan introducirse en las comunidades individuales. En este sistema de laissez-faire podria resultar que, aunque están permitidas, no hay instituciones "capitalistas" realmente funcionando; o bien, que algunas comunidades las tienen $y$ otras no o que algunas comunidades tienen algunas de ellas, o lo que usted quiera (p. 308).

17. P. Kropotkin, entrada anarqua de la edición de 1905 de la Enciclopedia Británica, en Obras, Barcelona, Anagrama, pp. 38-39.

18. Ibid, p. 39.

\title{
Las libertades inseparables, o razones y dudas del neoliberalismo
}

\author{
PEDRO SCHWARTZ \\ Universidad Autónoma de Madrid
}

Durante una breve época, que casi coincide con la década de 1980 , parecio a algunas mentes superficiales y supersticiosas que el ideario basado en la conjunción de las libertades personales, políticas, sociales y econórmicas, ideario que podríamos llamar "liberalismo ortodoxo", o «liberalismo de mercado", iba a triunfar necesaria y definitivamente. Incluso aparecio un escritor hegeliano, Francis Fukuyama, que se hizo famoso anunciando el final de 